\title{
TI.4.1
}

\section{CACTI Charter}

- PDF: CACTI Charter.pdf

- Text: TI.4.1.CACTI-Charter-Text

\begin{tabular}{|l|l|}
\hline More Information \\
\hline Repository ID & TI.4.1 \\
\hline Persistent URL & http://doi.org/10.26869/TI.4.1 \\
\hline Title & CACTI Charter \\
\hline Authors & Tom Barton \\
\hline Sponsor & Internet2 \\
\hline Review & Consultation for CACTI Charter \\
\hline Status & Preserve \\
\hline Publish Date & January, 2017 \\
\hline DOI & $10.26869 /$ TI.4.1 \\
\hline Signature & \\
\hline Deprecated & No \\
\hline Future Review & $2 / 1 / 2018$ \\
\hline Supersedes & \\
\hline Format & PDF, Text \\
\hline Related Docs & \\
\hline Development Location & \\
\hline IP Framework & CC BY 4.0 \\
\hline Subject Tags & charter, cacti \\
\hline Notes & \\
\hline
\end{tabular}

